\title{
Mujeres, teletrabajo y estrategias de cuidados en el contexto de pandemia en Chile ${ }^{*}$
}

DOI: https://doi.org/10.18046/recs.i35.4879

\author{
Women, Telework, and Care Strategies \\ in the Pandemic Context of Chile
}

\section{Catalina Arteaga-Aguirre ${ }^{* *}$}

Universidad de Chile (Santiago de Chile)

\section{Valentina Cabezas-Cartagena ${ }^{* * *}$ \\ Universidad de Chile (Santiago de Chile)}

\section{Fernanda Ramírez-Cid ${ }^{* * *}$}

Universidad de Chile (Santiago de Chile)

\footnotetext{
*El presente artículo se basa en la investigación “Madres, Teletrabajo y Cuidados en Pandemia”, realizada entre los meses de septiembre de 2020 y junio de 2021. Contó con el apoyo de la Red de Investigación en Interseccionalidad, Género y Prácticas de Resistencia (Red Iger), Universidad de Chile, financiada por la Vicerrectoría de Investigación y Desarrollo de la misma universidad. Artículo de investigación recibido el 23.06.2021 y aceptado el 08.11.2021.

** Académica del Departamento de Sociología, Universidad de Chile. Sus líneas de investigación son: género, cuidados, trabajo femenino, experiencia social. Coordina la Red de Investigación en Interseccionalidad, Género y Prácticas de Resistencia de la Universidad de Chile. Correo electrónico: carteaga@uchile.cl ORCID: https://orcid.org/oooo-ooo2o365-5526

*** Licenciada en Sociología de la Universidad de Chile. Correo electrónico: valentinacabezas88@gmail.com ORCID: https://orcid.org/oooo-0oo2-1118-0524

**** Licenciada en Sociología de la Universidad de Chile. Correo electrónico: fernanda.ramirez.c@ug.uchile.cl ORCID: https://orcid.org/oooo-0oo2-8799-0072
} 


\section{Cómo citar/How to cite}

Arteaga-Aguirre, Catalina; Cabezas-Cartagena, Valentina; Ramírez-Cid, Fernanda (2021). Mujeres, teletrabajo y estrategias de cuidados en el contexto de pandemia en Chile. Revista CS, 35, 11-39. https://doi.org/10.18046/recs.i35.4879 


\section{Resumen}

La pandemia de COVID-19 ha impactado a la población en distintas dimensiones, aumentando las desigualdades. Además de los efectos en la economía y la salud, se observan transformaciones en la cotidianidad, como la masificación del teletrabajo. Este hecho ha implicado una convergencia del espacio doméstico y el laboral, afectando en gran medida a las madres trabajadoras, quienes han aumentado su carga de trabajo de cuidados, impactando sus rutinas y su salud mental. El principal objetivo del presente artículo es analizar las estrategias que desarrollan madres chilenas que teletrabajan, a partir del confinamiento, para enfrentar las tareas de cuidado y su trabajo remunerado. Mediante el análisis de 18 entrevistas semiestructuradas, se reconocen 5 principales estrategias de las mujeres en este contexto: organización ante la ausencia de control del tiempo; multiplicidad y simultaneidad de tareas; apoyo escolar y proximidad; respuesta a la sobrecarga laboral, y resistencia.

\section{PALABRAS CLAVE:}

pandemia COVID-19, teletrabajo, cuidados, maternidad, estrategias

The COVID-19 pandemic has impacted the world population in different dimensions and has increased inequalities in different countries. In addition to the effects on the economy and health, transformations in daily life are observed, such as the massification of telework. This has implied the convergence of the domestic and working spaces, significantly affecting working mothers, who have increased their care workload, and impacting their daily routines and mental health. The main objective is to analyze the strategies developed by Chilean mothers, who telework from confinement measures, to deal with the conflict between care tasks and paid work. Through the analysis of eighteen semi-structured interviews, five types of strategies adopted by women in this context of double presence and unequal distribution of tasks were recognized: organizational in the absence of time control, multiplicity and simultaneity of tasks, school support and proximity, work against overload, resistance.

\section{KEYWORDS:}

COVID-19 Pandemic, Telework, Care, Motherhood, Strategies 



\section{Introducción}

La actual pandemia por COVID-19 ha llevado a los gobiernos a decretar el confinamiento obligatorio para la mayor parte de las personas, lo que ha significado una reorganización de la vida al interior de los hogares. El cierre de los establecimientos educacionales y otros centros de cuidados, sumado al cambio a la modalidad de teletrabajo para quienes pueden ejercerlo, ha provocado el aumento del trabajo de cuidados al interior del hogar como consecuencia del encierro, dejando expuesta la importancia de estas actividades para la sostenibilidad de la vida y la poca visibilidad que tiene este sector en las economías de la región (Comisión Económica para América Latina y el Caribe, 2020). Ante esta situación, las mujeres teletrabajadoras han enfrentado mayormente el desafío de compatibilizar sus tiempos de trabajo remunerado, de trabajo doméstico y de cuidados. Producto del encierro, ha aumentado la carga de este trabajo no remunerado, sobre todo cuando hay presencia de hijos/as menores de edad (Del Boca; Oggero; Profeta; Rossi, 2020). Este contexto acentúa un problema que venía desde antes de la crisis sanitaria: la sobrecarga de las mujeres que trabajan de forma remunerada y que, además, deben asumir las tareas de su hogar, trabajo que no es asumido como tal, sino como algo natural de las mujeres (Brenes, 2020). Por otro lado, no se han visto apoyos ni responsabilización gubernamental e institucional para enfrentar este incremento de trabajos de cuidados en los hogares. En ese contexto, la pandemia ha incrementado la crisis de los cuidados y la brecha de género (Ramacciotti, 2020), obligando a las mujeres a generar estrategias para enfrentar la crisis y los cuidados que anteriormente se encontraban externalizados.

La pregunta que guía el presente artículo es cuáles son las estrategias que desarrollan las madres de hijos/as menores de 14 años, que teletrabajan de forma remota, para llevar a cabo las tareas de cuidado, así como su trabajo remunerado, en el contexto de las medidas sanitarias frente a la pandemia. Asimismo, indagamos en las posibilidades de resistencia de las mujeres, a los mandatos de ser madre, mujer y trabajadora. Para responder estas interrogantes, desarrollamos una metodología cualitativa a través de entrevistas semiestructuradas en modalidad online, a mujeres teletrabajadoras de distintas ocupaciones, con hijos/as menores de 14 años.

De esta forma, se busca aportar en visibilizar la compleja situación que han debido enfrentar las mujeres trabajadoras durante la pandemia desde la vereda de los cuidados, algo que ha sido invisibilizado durante este particular contexto. Además, el análisis aporta desde un enfoque cualitativo, reconstruyendo las experiencias y relatos de un grupo poco analizado en los estudios disponibles. 


\section{Antecedentes}

\section{Cuidados en pandemia}

La situación de los cuidados en contexto de pandemia ha sido retratada por diversas encuestas de uso de tiempo en distintos países, las cuales muestran el aumento de las tareas de cuidado y su desigual distribución al interior del hogar (Boccardo; Andrade, 202O; Cadem, 202O; Ipsos, 2020). Entre las cifras que destacan, se encuentra que el $67 \%$ de las trabajadoras de servicios públicos sienten que el trabajo de cuidados aumentó durante este escenario, e indican que estas tareas son llevadas a cabo principalmente por ellas, ya que el 70,3\% de las encuestadas se declara la principal responsable de las labores domésticas, frente al 21,9\% de hombres (Boccardo; Andrade, 2020).

De acuerdo con datos de encuestas realizadas en el país, en cuanto a actividades que más se realizan y su distribución, se evidencia también una amplia desigualdad de género. En tareas de cocinar, hacer el aseo, lavar la ropa y dedicarse al cuidado de niños, todas tienen mayor presencia femenina. Por su lado, si bien los hombres también declaran hacer estas tareas, la que tiene mayor presencia masculina es cocinar, donde el $30 \%$ de ellos declara hacerlo, frente al $56 \%$ de ellas. El cuidado de niños y niñas es la actividad que menos hacen los hombres, con solo un $18 \%$, versus un $51 \%$ de mujeres que dice dedicarse a eso (Ipsos, 2020'). En la misma línea, otra encuesta señala que el acompañamiento escolar en casa también es atendido mayormente por mujeres: el $67 \%$ de madres declara acompañar a sus hijos/as en la educación en casa todos los días de la semana, versus el $43 \%$ de padres que declara hacerlo (Palma et al., 2020²). Con relación al uso del tiempo, las encuestas también dan cuenta de la desigual distribución de género. Según Cadem (2020) las mujeres dedican, en promedio, 14 horas al día a actividades tales como cuidar niños, labores domésticas (como aseo y cocinar) y ayudar a los niños en sus estudios, lo que ha

\footnotetext{
1. El Informe Claves 2O2O, edición abril: Chile en tiempos de coronavirus (Ipsos, 2O2O) fue una colaboración entre Ipsos y La Tercera, aplicada entre marzo y abril de 2020 sobre el panel web nacional de Ipsos, a hombres y mujeres mayores de 18 años de todas las regiones, con una muestra de 599 casos a nivel nacional, y diseño no probabilístico de muestreo por cuotas.

2. "Vida en Pandemia" es un estudio longitudinal que, en su cuarto informe, muestra la desestabilización y reorganización de la vida doméstica durante la crisis del COVID-19. La encuesta analizada fue realizada entre el 13 y el 17 de julio de 202O, en línea, a una muestra heterogénea de 2552 personas, con diseño no probabilístico por cuotas.
} 
aumentado enormemente en el actual contexto. Por su parte, los hombres dedican 8 horas diarias a estas labores (Cadem, 20203).

Estos datos evidencian la agudización de la división sexual del trabajo, que esencializa el trabajo del cuidado de la familia y del hogar en las mujeres. Ante esta situación, un $31 \%$ de las mujeres indica sentirse agobiada por la cantidad de tareas que debe realizar al estar en casa por las medidas sanitarias frente al coronavirus, mientras que solo un $17 \%$ de los hombres manifiesta ese agobio (Ipsos, 2020). Esta situación es causada, principalmente, por el fenómeno de la doble presencia, que demanda la atención tanto en las tareas del hogar y de cuidado, como en el teletrabajo. El estudio de Boccardo y Andrade (2020) señala que, en el sector público, $62,3 \%$ de las mujeres piensan siempre o casi siempre en las exigencias domésticas y familiares durante el trabajo (versus el 44,0 \% de los hombres) y son ellas también quienes indican, en mayor medida que sus pares masculinos, tener tareas del hogar que interfieren con su trabajo (26,6\% indica que siempre o casi siempre) y tener que interrumpir su trabajo por el cuidado de algún miembro de su hogar $(26,1 \%)$. Esta doble presencia se da en cualquier modalidad que lleve a cabo la trabajadora -teletrabajo total, teletrabajo mixto o modalidad presencial-, aunque alcanza mayores niveles cuando se encuentra trabajando totalmente desde su casa (Boccardo; Andrade, 2020).

\section{Mujer y teletrabajo durante la crisis sanitaria}

El teletrabajo puede definirse como "el uso de tecnologías de la información y las comunicaciones -como teléfonos inteligentes, tablets, laptops y ordenadores de sobremesa- para trabajar fuera de las instalaciones del empleador" (Organización Internacional del Trabajo, 2019:1). Durante el confinamiento, se ha instalado como alternativa, lo cual ha significado, para las mujeres con hijos/as, la compatibilización forzada del trabajo remunerado con la vida familiar, asumiendo problemas como las distracciones constantes por miembros de su familia, la adaptación de sus horarios a las necesidades de otros, la superposición de tareas, el no tener tiempos específicos de trabajo (Hilbrecht; Shaw; Johnson; Andrey, 2013), jornadas laborales más largas y aumento de carga laboral (Pérez; Gálvez, 2009). A esto se suman nuevas variables producto de la pandemia, tales como la presencia de hijos/as en casa por el cierre

3. El estudio "El Chile que viene: diferencias de género frente al covid-19", de septiembre de 2020, fue realizado por Cadem, mediante encuestas autoadministradas aplicadas a través de entrevistas web al panel Cadem Online, con una muestra de 2597 personas, entre hombres y mujeres mayores de 18 años, de todos los niveles socioeconómicos y todas las regiones del país. Fue aplicada entre el to y el 14 de septiembre de 2020 . 
de escuelas y centros de cuidado, viéndose aumentadas sus responsabilidades. Además, la sobrecarga se ve intensificada por la condición de aislamiento físico, en la cual los lugares y tiempos de trabajo remunerado son los mismos que de trabajo no remunerado.

También, se debe tener en cuenta que esta situación se debió aceptar y llevar a cabo de forma rápida e improvisada, desde marzo de 2020 en el caso de Chile 4 , obligando a las mujeres a organizarse en un escenario de crisis, priorizando, muchas veces, el trabajo doméstico por la inmediatez de sus necesidades, mientras el remunerado es trasladado al horario nocturno, imposibilitando el tiempo de ocio y descanso (Brenes, 2020).

La superposición de espacios, la organización obligada de sus tiempos de trabajo, alimentación, ocio y convivencia, y el asumir actividades y habilidades nuevas como el ejercicio docente para ayudar a hijos/as en edad escolar, trajo consecuencias negativas para estas mujeres, tanto a nivel psicológico, como físico, anímico y social (Brenes, 2020). Se ha detectado que las madres que teletrabajan han reportado mayores niveles de ansiedad, sentimiento de soledad y se deprimen más que los padres teletrabajadores (Lyttelton; Zang; Musick, 2020).

Por otro lado, la edad de los hijos/as afecta la carga a la que se deben enfrentar sus madres. Los/as menores de 10 años son más demandantes para padres y madres, mientras que estudiantes de escuela primaria requieren más apoyo en sus estudios, lo que es suplido mayormente por sus madres; son las mujeres con títulos universitarios quienes dedican más tiempo a asistir en sus estudios a sus hijos/as. En hijos/as mayores de 15 años, la participación de sus padres es menor y son las mujeres con hijos/as entre o y 5 años las que declaran en mayor medida un exceso de responsabilidades domésticas (Del Boca et al., 2020).

En Chile, de acuerdo con el estudio Cadem (2020), de quienes se encuentran trabajando, es mayor el porcentaje de mujeres que de hombres que han asumido la

4. El 3 de marzo de 2020 llegó el primer caso de COVID-19 a Chile (“Ministerio de Salud...", 2O2O), al que rápidamente se le fueron sumando más casos. Tras eso, el Gobierno decidió, el 15 de marzo, suspender las clases en jardines infantiles, colegios municipales, subvencionados y particulares ("Presidente anuncia suspensión...”, 2020). Tres días después se decretó el estado de excepción constitucional de catástrofe en todo el país ("Presidente Piñera decreta...", 2020), lo que permitió establecer el toque de queda y las primeras cuarentenas totales. Los establecimientos escolares se mantuvieron cerrados, incorporando únicamente clases a distancia, ya fuera en línea u otro sistema, hasta julio de 2020, cuando se permitió, por primera vez, a una escuela en Chile continental volver a clases presenciales (“Liderar en tiempos...”, 2020). De todas maneras, esto no significó una vuelta definitiva a clases presenciales, la cual será obligatoria desde marzo de 2022 (“A partir de marzo...", 2021), permitiendo, por el momento, que los establecimientos mantengan clases de forma totalmente remota o en formato híbrido (combinando clases presenciales y virtuales). 
modalidad de teletrabajo durante la crisis sanitaria5. En este panorama, las mujeres reconocen, en mayor medida que los hombres, que un aspecto que dificulta el teletrabajo es tener que compatibilizarlo con las tareas del hogar (Cadem, 2020). En sintonía con lo anterior, de acuerdo con un estudio enfocado en la situación de trabajadores/as de servicios públicos, se señalan como dificultades para las trabajadoras el no contar con espacio propio para trabajar, trabajar con insumos no adecuados para el trabajo (no contar con escritorio o silla adecuada) y la casi nula posibilidad de desconexión tanto del trabajo remunerado como de las exigencias domésticas (Boccardo; Andrade, 2020).

A continuación, se abordan los conceptos centrales que nos orientan en el análisis, para luego describir la estrategia metodológica llevada a cabo y los resultados de la investigación.

\section{Conceptos teóricos: cuidados y estrategias de cuidado en contexto de crisis}

\section{Cuidados}

El trabajo de cuidados ha sido definido por distintos/as autores/as, para dar cuenta de las actividades orientadas al cuidado de la vida en sus aspectos tanto físicos como emocionales. Carrasco (2013) define este trabajo como aquel realizado desde los hogares, orientado al cuidado de las personas a lo largo del ciclo vital, con las dependencias específicas que implica cada etapa de la vida y en sus distintas dimensiones: cuidados directos afectivos y emocionales, cuidado del entorno, cuidado de las relaciones, cuidados en la salud y producción de determinados bienes y servicios.

Por su parte, Batthyány (como se citó en Giaconi, 2021: 1) plantea que "el cuidado puede ser entendido como un trabajo, una relación interpersonal, y como una responsabilidad socialmente construida que se inscribe en contextos sociales y económicos particulares". Además, el trabajo de cuidados se entiende como un trabajo invisible, devaluado cultural y económicamente (Hatton, 2017). Según Glenn (como se citó en Giaconi, 2021: 1), esta devaluación y poca visibilidad "genera que el cuidado quede relegado y sea responsabilidad de aquellos grupos que carecen de poder y de estatus político, económico y social, siendo provisto principalmente por grupos desfavorecidos, entre ellos, las mujeres".

5. Con base en quienes se encuentran con trabajo, el $44 \%$ de las mujeres está teletrabajando, frente al $23 \%$ de hombres. 
En las últimas décadas, el cuidado ha tomado relevancia académica y política por la llamada crisis de los cuidados, generada por el aumento de personas en situación de dependencia y la disminución de personas que realicen esta labor, por los cambios demográficos, aumento de la esperanza de vida, cambios familiares e ingreso de la mujer al trabajo remunerado (Federici, 2009).

\section{Estrategias de cuidado}

Aunque las actividades de cuidado se han naturalizado, la situación de crisis y el contexto de encierro de las mujeres en la actualidad han fomentado el desarrollo de una multiplicidad de actividades en el mismo espacio y al mismo tiempo. Ello puede ser abordado desde la noción de estrategias de cuidados (Batthyány; Genta; Scavino, 2017), las cuales se consideran como elecciones llevadas a cabo por quienes integran los hogares, y se ven influidas por factores estructurales (división sexual del trabajo, posición de clase, entre otras) y culturales (roles de género respecto a tareas de cuidados), que determinan la distribución de las tareas entre los distintos miembros involucrados.

Estas estrategias suelen desarrollarse en la esfera privada, al ser consideradas como un problema exclusivo de las familias y de las madres, quienes deben compatibilizar su empleo con labores de cuidado. El apoyo de la familia extensa o de personas externas puede estar presente, pero son principalmente las madres las encargadas de distribuir los cuidados, siendo muy limitado el apoyo desde políticas públicas que consideren este un problema social (Tobío, 1998).

Dichos conceptos serán puestos en tensión, de cara a la experiencia de mujeres que han debido confinarse en el espacio doméstico, a partir de las medidas frente al COVID-19 y desarrollar su trabajo en modalidad a distancia, con hijos/as pequeños a su cuidado.

\section{Metodología}

La investigación se orientó por la metodología cualitativa, de acuerdo con el objetivo de la investigación, dirigida a conocer las estrategias de madres teletrabajadoras en contexto de confinamiento, a partir de las medidas sociosanitarias frente a la pandemia. Esta metodología se centra en conocer y comprender las perspectivas de los sujetos, sus sentidos, sus significados y sus experiencias. Emplea métodos de análisis y de comprensión sensibles al contexto en el que los datos son producidos. Analiza una práctica real, situada, y desarrolla un proceso interactivo, en el que intervienen el/la investigador/a y los participantes (Vasilachis, 2006). En ese marco, se opta 
por diseñar y desarrollar entrevistas semiestructuradas (Flores, 2009), orientadas a llevar a cabo una conversación entre las investigadoras y las trabajadoras entrevistadas, sobre temas definidos en una pauta para ese fin, de manera relativamente flexible. Se realizaron un total de 18 entrevistas, acorde a los siguientes criterios de selección de la muestra: 1) mujeres madres que vivan con hijos/as de hasta 14 años, y 2) mujeres teletrabajadoras a partir del confinamiento de marzo de 2020, en distintas modalidades

Se estableció un muestreo intencional (Izcara, 2007) de mujeres con trabajo estable en modalidad de teletrabajo por la pandemia, con diversidad de ocupaciones e ingresos, siendo la mayoría profesionales de clase media alta, aunque también se incluyeron mujeres trabajadoras del área de servicios. Se entrevistaron mujeres del sector educativo (profesoras de enseñanza básica, media y universitarias); secretarias; profesionales del área empresarial, del comercio y finanzas; profesionales del sector público, y periodistas. Todas las entrevistadas son madres de hijos/as de hasta 14 años y viven con ellos/as. Sus nombres han sido cambiados para proteger su identidad. Las principales características de las entrevistadas se incluyen en el Cuadro 1.

\begin{tabular}{|c|c|c|c|c|c|c|}
\hline \multicolumn{2}{|c|}{ CUADRO 1} & \multicolumn{5}{|c|}{ Caracterización de las entrevistadas } \\
\hline $\begin{array}{l}\text { No. } \\
\text { entre- } \\
\text { vista }\end{array}$ & Nombre & Edad & Ocupación & $\begin{array}{l}\text { Modalidad de } \\
\text { teletrabajo }\end{array}$ & $\begin{array}{c}\text { Número y } \\
\text { edades de hijos/ } \\
\text { as en el hogar }\end{array}$ & $\begin{array}{c}\text { Otros/as } \\
\text { integrantes del } \\
\text { hogar y edades }\end{array}$ \\
\hline $\mathrm{O} 1$ & Erika & 43 & $\begin{array}{c}\text { Docente } \\
\text { universitaria }\end{array}$ & $\begin{array}{l}\text { Teletrabajo } \\
\text { total }\end{array}$ & $\begin{array}{l}1 \text { hijo ( } 7 \text { años) } \\
1 \text { hija (4 años) }\end{array}$ & Pareja (42 años) \\
\hline $\mathrm{O} 2$ & Karla & 29 & $\begin{array}{c}\text { Docente } \\
\text { universitaria } \\
\text { y asistente de } \\
\text { investigación }\end{array}$ & $\begin{array}{l}\text { Teletrabajo } \\
\text { mixto }\end{array}$ & 1 hija (10 años) & Pareja (36 años) \\
\hline $\mathrm{O} 3$ & Laura & 42 & $\begin{array}{l}\text { Ingeniera } \\
\text { comercial }\end{array}$ & $\begin{array}{l}\text { Teletrabajo } \\
\text { total }\end{array}$ & $\begin{array}{l}1 \text { hijo (12 años) } \\
1 \text { hija (10 años) }\end{array}$ & No \\
\hline $\mathrm{O} 4$ & Natalia & 51 & Profesora & $\begin{array}{l}\text { Teletrabajo } \\
\text { total }\end{array}$ & 1 hija (12 años) & Pareja (41 años) \\
\hline 05 & Pamela & 42 & Secretaria & $\begin{array}{l}\text { Teletrabajo } \\
\text { total }\end{array}$ & 1 hijo (8 años) & No \\
\hline 06 & Sandra & 30 & Secretaria & $\begin{array}{l}\text { Teletrabajo } \\
\text { mixto }\end{array}$ & 1 hijo (4 años) & Pareja (35 años) \\
\hline 07 & $\begin{array}{l}\text { María } \\
\text { José }\end{array}$ & 37 & Profesora & $\begin{array}{l}\text { Teletrabajo } \\
\text { total }\end{array}$ & 1 hijo (5 años) & $\begin{array}{l}\text { Padre (65 años) } \\
\text { Madre (59 años) }\end{array}$ \\
\hline
\end{tabular}




\begin{tabular}{|c|c|c|c|c|c|c|}
\hline o8 & Carola & 27 & Profesora & $\begin{array}{l}\text { Teletrabajo } \\
\text { total }\end{array}$ & 1 hijo (4 años) & No \\
\hline 09 & Susana & 40 & Socióloga & $\begin{array}{c}\text { Teletrabajo } \\
\text { total }\end{array}$ & 1 hija (9 años) & Pareja (41 años) \\
\hline 10 & Daniela & 35 & $\begin{array}{c}\text { Trabajadora } \\
\text { social }\end{array}$ & $\begin{array}{l}\text { Teletrabajo } \\
\text { mixto }\end{array}$ & $\begin{array}{l}1 \text { hija (18 años) } \\
1 \text { hija ( } 9 \text { años) }\end{array}$ & Madre (56 años) \\
\hline 11 & Pía & 46 & Periodista & $\begin{array}{l}\text { Teletrabajo } \\
\text { total }\end{array}$ & $\begin{array}{l}1 \text { hija (9 años) } \\
1 \text { hija (11 años) }\end{array}$ & Pareja (44 años) \\
\hline 12 & Fabiola & 54 & $\begin{array}{l}\text { Coach, } \\
\text { freelance }\end{array}$ & $\begin{array}{c}\text { Teletrabajo } \\
\text { total }\end{array}$ & $\begin{array}{l}1 \text { hija (16 años) } \\
1 \text { hijo (13 años) }\end{array}$ & Pareja (56 años) \\
\hline 13 & Fernanda & 31 & $\begin{array}{l}\text { Jefa de } \\
\text { servicio al } \\
\text { cliente }\end{array}$ & $\begin{array}{l}\text { Teletrabajo } \\
\text { total }\end{array}$ & $\begin{array}{c}1 \text { hijo (10 años) } \\
1 \text { hija (1 año) }\end{array}$ & $\begin{array}{l}\text { Pareja ( } 37 \text { años) } \\
\text { Prima ( } 50 \text { años, } \\
\text { iba a ayudar por } \\
\text { períodos) }\end{array}$ \\
\hline 14 & Camila & 48 & Secretaria & $\begin{array}{c}\text { Teletrabajo } \\
\text { mixto }\end{array}$ & 1 hija (10 años) & Pareja (42 años) \\
\hline 15 & Diana & 44 & $\begin{array}{c}\text { Oficial de } \\
\text { cumplimiento }\end{array}$ & $\begin{array}{c}\text { Teletrabajo } \\
\text { total }\end{array}$ & $\begin{array}{l}1 \text { hijo (10 años) } \\
1 \text { hija (6 años) }\end{array}$ & No \\
\hline 16 & $\begin{array}{l}\text { Cons- } \\
\text { tanza }\end{array}$ & 46 & Arquitecta & $\begin{array}{l}\text { Teletrabajo } \\
\text { total }\end{array}$ & $\begin{array}{l}1 \text { hija (15 años) } \\
1 \text { hija (13 años) } \\
1 \text { hijo (9 años) }\end{array}$ & Pareja (47 años) \\
\hline 17 & Alicia & 38 & $\begin{array}{l}\text { Tecnóloga } \\
\text { médica }\end{array}$ & $\begin{array}{l}\text { Teletrabajo } \\
\text { mixto }\end{array}$ & 1 hija (3 años) & Pareja (38 años) \\
\hline 18 & Cristina & 46 & Periodista & $\begin{array}{l}\text { Teletrabajo } \\
\text { total }\end{array}$ & $\begin{array}{l}1 \text { hijo (14 años) } \\
1 \text { hija (11 años) }\end{array}$ & Pareja (43 años) \\
\hline
\end{tabular}

Fuente: elaboración propia.

Se diseñó una pauta de entrevista semiestructurada, en torno a los temas: caracterización del empleo y modalidad de teletrabajo; organización del teletrabajo; actividades de cuidados; experiencia socioemocional; cuidado infantil; acceso a apoyos institucionales y redes. Se estableció un consentimiento informado, aprobado en la sesión de entrevistas. Estas fueron realizadas vía Zoom, con una duración promedio de 1 hora y 15 minutos, desarrolladas entre noviembre de 2020 y mayo de 2021.

Para sistematizar la información, se trabajó con la codificación de la teoría fundamentada (Strauss; Corbin, 2002), en las fases de codificación abierta y axial, a partir del establecimiento de códigos y categorías. Se llevó a cabo un análisis principalmente inductivo, el cual fue discutido con la revisión teórica llevada a cabo en la fase previa al desarrollo de las entrevistas, incidiendo en nuevas lecturas y reflexiones interpretativas. 


\section{Resultados}

Si bien las mujeres entrevistadas tienen ocupaciones diversas, comparten algunas condiciones transversales en relación con las características del empleo femenino en Chile, las cuales se han intensificado a partir de las medidas sanitarias implementadas desde marzo de 2020 en el país. La inserción de las mujeres en el mercado del trabajo es relativamente tardía y una de las más bajas de América Latina, aunque en aumento en los años anteriores a la pandemia (53,3 \%) (Instituto Nacional de Estadísticas [INE], 2021). Asimismo, se concentran en empleos de menor calificación y remuneración. En la actualidad, el impacto de la pandemia ha sido mayor sobre el empleo de las mujeres debido a la brecha histórica que han registrado (INE, 2021). Junto a este impacto, las medidas sanitarias implicaron un cambio del lugar de trabajo a partir de las cuarentenas, lo que incidió en mayor medida en las mujeres que en los hombres.

Las mujeres entrevistadas comparten ciertas características en las formas de organización del trabajo, así como problemas y estrategias para la coexistencia de las distintas responsabilidades domésticas y laborales. Existen, sin embargo, algunas diferencias derivadas de las características socioeconómicas, lo cual impacta en las condiciones materiales de trabajo, acceso a espacios y de apoyo doméstico, como se mostrará en el análisis realizado.

Para comprender las tensiones entre los distintos espacios que conviven actualmente en el hogar, así como las estrategias desplegadas por las madres teletrabajadoras para hacer frente a estas, se describirán en primer lugar las actividades de cuidados que ellas realizan a diario en el contexto de encierro, la forma en que se han organizado y la división de tareas presente en el hogar durante este período. Posteriormente, se ahondará en cinco estrategias detectadas en las experiencias de las mujeres: de organización ante ausencia de control del tiempo; de multiplicidad y simultaneidad de tareas; de apoyo escolar y proximidad; laborales frente a la sobrecarga, y de resistencia.

\section{Aumento de tareas de cuidados y trabajo remunerado en pandemia}

A pesar de las diferencias, todas las entrevistadas señalan haber aumentado la multiplicidad de tareas de cuidado. Esto se evidencia en sus testimonios, donde mencionan que al estar más tiempo habitada la casa, las actividades de orden y limpieza se hacen necesarias con mayor frecuencia. Además, al coincidir el lugar de vivienda y trabajo, varias declaran que el desorden puede interferir en su concentración: "lo que me quita más tiempo..., como ordenar porque soy estresada, no puedo estar cuando 
está todo muy desordenado, porque si está todo muy desordenado [me] empieza a tiritar el ojo, así que yo ordeno" (Susana, comunicación personal, 20.01.2021).

El aumento de tareas en el hogar ha sido asumido mayoritariamente por las madres, incluso en los hogares donde los padres también teletrabajan. En el contexto de encierro, son ellas quienes asumen principalmente las tareas de cuidado, por la dificultad de acceder a redes de apoyo familiar externas, como abuelas, tías, hermanas, que antes estaban disponibles para ayudar en estas labores. A lo anterior, se suma la carga de tener que asistir a hijos/as con las clases a distancia, lo que implica un mayor nivel de acompañamiento e involucramiento por parte de madres y padres, en comparación con las clases presenciales, como veremos más adelante, aunque esto también depende de la autonomía y edad de los hijos/as.

Las múltiples labores que realizan las mujeres a lo largo del día dan la sensación de agobio y de que nunca terminan de realizar las tareas del hogar: "ha sido igual una locura porque siento que nunca termino de ordenar y tengo varias cosas, y me gusta que todo tenga su lugar. Entonces me paso el día ordenando, la verdad, y nunca termino de ordenar" (Carola, comunicación personal, 06.01.2021).

A pesar de que la gran mayoría de nuestras entrevistadas vive con el padre de sus hijos/as, no hay una percepción de gran responsabilidad en las actividades domésticas de su parte. El apoyo escolar es donde se menciona un mayor involucramiento por parte de las parejas, aunque en general señalan que son ellas quienes más apoyan a sus hijos/as. Algunos padres se involucran con ciertas asignaturas, o los fines de semana, mientras en otros hogares esta tarea es exclusiva de las madres; aunque, a veces, el involucramiento genera conflicto entre los padres:

Y la verdad es que igual mi marido no es como yo, es como más relajado, entonces si él no quería, no la hacía [la tarea], y había semanas en que ellos no hacían ninguna tarea, entonces se me juntaba con lo de la semana que yo estaba acá en la casa, y tenía más tarea, entonces ya como que eso me estresaba un poco. (Sandra, comunicación personal, 29.12.2O2O)

Respecto al panorama laboral y carga de trabajo remunerado de las entrevistadas, se relata cierta improvisación, producto de lo abrupto del comienzo del trabajo remoto. En algunos casos -los menos-, por lo poco que podían hacer a distancia, la carga laboral bajó. Sin embargo, en la mayoría de los testimonios se menciona una sensación de inestabilidad respecto a la carga de trabajo que varía de acuerdo con el día, aunque prima la sensación de una mayor responsabilidad a nivel general.

Entremedio que uno está trabajando, echas la ropa en la lavadora, entremedio que estás revisando, que estoy leyendo un decreto, pongo la aspiradora que aspire (...) y entreme- 
dio que estoy en la mañana, me paraba como a las 13:00, y en ese rato me paraba a ver: “ya, y ahora, ¿qué vamos a almorzar?”. (Susana, comunicación personal, 20.01.2021)

La mayoría de las entrevistadas no cuenta con un horario de trabajo remunerado delimitado, no trabaja en un horario fijo y declara que en la modalidad en línea ha extendido su jornada de trabajo, donde es común que se les exija disponibilidad más allá de su horario formal de trabajo, el cual no se respeta.

Yo siento que nos perjudicó el tema de trabajar online, porque teníamos nosotros un horario de trabajo, de 8:30 a 18:oo, otros días, por ejemplo, yo tenía el jueves que salía a las 12:30 y, de repente, me encontraba con que, de modalidad online tenía una reunión de apoderados el día jueves a las 18:oo, fuera de mi horario de trabajo, o los días viernes a las 18:0o, fuera de mi horario de trabajo. (Carola, comunicación personal, o6.01.2021)

A la mayoría les resulta difícil calcular el tiempo real de trabajo, pues se aprecia un desdibujamiento de los límites del horario. Algunas han expresado la sensación de no parar de trabajar y de hacerlo más que en modalidad presencial: el tiempo tradicional que se ocupaba en el desplazamiento y aquel destinado al descanso después de almorzar son ahora tiempos de trabajo.

Ya no me desplazaba, ya no almorzaba en el colegio, almorzaba acá, pero como terminaba de almorzar y estaba trabajando, y terminaba y seguía trabajando, y seguía trabajando, ¿cachai6? Entonces igual era como sin parar al principio, como no tenía muy claro el límite, pero también me parecía que era necesario, o sea, yo al menos entré en otra lógica. (Natalia, comunicación personal, 28.12.2020)

En otros casos, el miedo a quedar sin trabajo llevó a que las mismas entrevistadas se ofrecieran a hacer más en su empleo y aumentaran su carga laboral.

Me pasó que (...) nosotros que somos como consultores, un poco de... con miedo a que nos fueran a cancelar el contrato, empezamos a ofrecerle más cosas al cliente (...), para que nos necesitaran. Entonces empezaron a pasar más y más trabajo. (Laura, comunicación personal, 10.12.202O)

Las modalidades de teletrabajo varían en los casos analizados, evidenciando dos tipos: teletrabajo completo y teletrabajo mixto (semana por medio, o algunos días al mes presencial). Algunas han debido volver al trabajo presencial por algunos días, y agradecen recuperar el espacio propio.

6. Modismo chileno: ¿entiendes? 
Bueno, yo creo que también me ayudó esto de empezar, de volver a trabajar, o sea, esto de tener mi espacio de nuevo, que es superimportante y es algo que no se puede hacer en la casa, o sea, yo creo que es casi imposible, no... ese espacio por último de escuchar el silencio de uno mismo mientras uno va caminando hacia el trabajo no existía, po y pa mí siempre ha sido supernecesario. (Karla, comunicación personal, 03.12.2O2O)

El espacio de trabajo y el acceso a tecnología varían entre las entrevistadas, lo que da cuenta de diferencias socioeconómicas. No obstante, se dan algunas coincidencias: todas debieron adaptar algún lugar de la casa para teletrabajar, y casi todas trabajan en el mismo espacio en donde sus hijos/as tienen clases. Muy pocas trabajan en un espacio propio, como oficina con escritorio y silla correcta, pues casi todas trabajan en comedor o dormitorio con sus hijos/as a la vista.

Yo adapté un lugar como para hacer mis cosas del trabajo, obviamente no son las óptimas porque no tengo una silla ergonómica, es una silla común y corriente del comedor, la mesa es una mesa de estas de pícnic, porque no tengo un escritorio. (Pamela, comunicación personal, 28.12.2020)

Tuve que comprar, compré todo. De la empresa me pasaron celular, notebooky, bueno, la impresora era mía y tuve que comprar la pantalla, por comodidad mía compré el teclado, compré lámpara, compré la silla, compré el escritorio y la pizarra me la regaló mi esposo. (Fernanda, comunicación personal, 20.04.2021)

En cuanto a la evaluación de sus condiciones y modalidad de trabajo, hay diferencias importantes entre las entrevistadas. Las profesoras -3 de colegio y 2 universitarias- son las que más revelan problemas y sobrecarga de trabajo por esta nueva modalidad: el no ver a sus alumnos, que algunos no asistan a clases y la corrección de trabajos en línea implican más trabajo y presión que antes. Probablemente, esta percepción se relaciona con el cierre de las escuelas desde marzo de 2020 y la ausencia de contacto directo con los/as alumnos/as.

El trabajo es dos o tres veces más, en el caso mío como profesora, no sé otras áreas de teletrabajo, pero en el caso mío, sí; se triplicó, cuadruplicó mucho. Cosas tan simples como las que te explicaba que cuando uno está en la sala de clase, detectas un error, paras la clase, corriges, los 45 te entienden y listo. Ahora no, tienes que ir uno por uno corrigiendo; es demasiado. (María José, comunicación personal, 04.01.2021)

Hacemos guías, hacemos presentaciones de PowerPoint, estuvimos trabajando también con libros, y lo que hacíamos era hacer audiolibros, cosa que los chicos pudieran 
ver el video con el texto y a la vez nosotros irles narrando; hacíamos actividades, juegos online, juegos relacionados con vocabulario, con el mismo libro, con la materia que estábamos pasando, y la clase consistía básicamente en ir mostrando los ejercicios. (Carola, comunicación personal, 06.01.2021)

Como se ha señalado, se evidencian diferencias importantes al analizar las condiciones de trabajo con relación al nivel socioeconómico de las mujeres entrevistadas, ya que algunas cuentan con mejores espacios para trabajar, adquirieron mejor tecnología e incluso ayuda externa pagada para el cuidado de sus hijos/as y el hogar.

Gracias a que tuve nana ${ }^{8}$, porque o si no, no sé qué hubiera hecho, por suerte que tuve nana. Hay gente que su nana no pudo venirse [a quedarse a la casa], mi nana no tenía familia, entonces se pudo venir a mi casa no más. (Laura, comunicación personal, 10.12.2O2O)

Sin embargo, para la mayoría, su casa es señalada como un lugar poco tranquilo para trabajar, debido a las interrupciones de sus hijos/as y las múltiples tareas que cumplen en ella.

Pregunta: Y sobre estar en la casa, ¿tú dirías que es un lugar tranquilo para trabajar?

Respuesta: No, po, no, para nada [se ríe]. No, porque no puedes evitar hacer cosas de casa, no lo puedes evitar porque estás ahí. ¿Cómo vas a decir: "ya no, no voy a barrer, me voy a sentar aquí donde están todas las migas en el suelo”? No vas... porque además tienes que hacerlo tú misma. (Susana, comunicación personal, 20.01.2021)

\section{Estrategias de organización ante ausencia de control del tiempo}

Ante el aumento de tareas de cuidado asumidas por las mujeres, son ellas las que principalmente organizan sus tiempos para realizarlas y, además, cumplir con sus trabajos remunerados. Ello ha implicado un cambio importante en comparación a sus rutinas previas a la pandemia. En este contexto, las mujeres se enfocan primordialmente en las tareas que involucran necesidades básicas o la atención de situaciones urgentes, como las actividades relacionadas directamente con los hijos/as, que son las más mencionadas como impostergables, como alimentarlos/as e indicar sus responsabilidades y aseo personal, por ejemplo.

8. Trabajadora de casa particular. 
Terminábamos las clases a las 13:00, y la próxima conexión era a las 15:00, entonces en ese tiempo trataba de hacer lo esencial, más que nada, que era cocinar, porque si hay que priorizar para mí, el tema del aseo, de tener todo limpio, trapeado, aspirado, no es relevante, eso lo puedo hacer cuando tenga tiempo, pero me dedicaba a cocinar, a tener el almuerzo listo, a darle el almuerzo a mi hijo para después conectarme de nuevo. (Carola, comunicación personal, 06.01.2021)

La mayoría de las mujeres menciona que el almuerzo es la actividad que más les preocupa y les toma tiempo, por lo que deben planificarlo bien para cocinar dentro de sus tiempos libres de trabajo, para disponer de él al momento en que sus hijos/as tienen horario de colación. Ante la urgencia de atender a estas tareas, las entrevistadas mencionan que organizan su día para realizar lo urgente a determinada hora y de forma rutinaria, dejando muchas veces el trabajo remunerado en segundo plano, además de otras actividades como el orden, la limpieza y las compras, por ser consideradas secundarias: "de repente eran la 1:30, 2:0o de la mañana y yo todavía estaba avanzando, entonces decía: 'no, ya', y ahí me tuve que empezar como a organizar con eso, porque me pasaba" (Natalia, comunicación personal, 28.12.2O2O).

Fue harto porque me costaba encontrar el tiempo para trabajar tranquila, y lo que hacía en ese momento era delegar todo lo que tenía que hacer en el día, o sea, laboralmente, a mi tiempo libre, que era la noche. Entonces me quedaba en la noche o planificando o preparando material o poniendo las notas, porque era el momento en el que no me interrumpía nadie. (Carola, comunicación personal, 06.01.2021)

\section{Ante doble presencia: estrategias de multiplicidad y simultaneidad de tareas}

Una estrategia tradicional de las mujeres para hacer frente a sus múltiples actividades se relaciona con la multiactividad, aunque, en el contexto de confinamiento, se traslapan actividades con distintos tipos de exigencias: aquellas de limpieza, alimentación, cuidados, paralelamente a las que requieren habilidades específicas relacionadas con sus empleos remunerados. Este fenómeno fue identificado por Laura Balbo, a finales de la década de 1970, como doble presencia, refiriéndose a la situación que suelen enfrentar las mujeres al insertarse en el mundo laboral, sin que eso signifique dejar de lado su papel de principales cuidadoras (Carrasquer, 2013). Su presencia es requerida de forma simultánea en su trabajo asalariado y en el ámbito doméstico-familiar (Moreno; Moncada; Llorens; Carrasquer, 2010).

La realización de ambas tareas se traduce en que "durante el tiempo de trabajo remunerado, la persona debe gestionar sus responsabilidades domésticas y, durante 
el tiempo privado, debe organizar o gestionar de alguna forma sus responsabilidades profesionales" (Ruiz; Pullas; Parra; Zamora, 2017: 35). Sin embargo, en la situación de confinamiento, tanto los espacios como los tiempos de lo privado y laboral se han superpuesto, por lo que Moré (2020) refiere a una doble presencia simultánea en tiempos de coronavirus, lo que lleva a las mujeres a intensificar la simultaneidad como estrategia para abordar las distintas exigencias.

Porque ya ser una mamá trabajadora es doble pega, y además estarlo haciendo al mismo tiempo del cuidado de los hijos es una pega tremenda (...). No es como que uno pueda dejar el cuidado de los hijos y enfocarse solo en la pega. (Carola, comunicación personal, 06.01.2021)

De esta forma, las entrevistadas mencionan comúnmente que hacen al menos dos cosas al mismo tiempo y, además, están constantemente pendientes de otras actividades o procesos mientras trabajan.

En el caso de mi hija, no es una niña inquieta ni porfiada, pero sí demanda atención. Entonces lo que a mí me ha pasado es que uno está, pero no está, estoy ahí, pero estoy en reunión, entonces estoy con audífono, por lo tanto, ella no me puede hablar porque no la escucho, o estoy sin audífono, pero estoy contestando cosas, estoy manejando presupuesto. (Susana, comunicación personal, 20.01.2021)

Además, se debe tener en cuenta que se hace referencia a doble presencia y no a doble jornada, porque se considera que lo que conecta el trabajo remunerado y las actividades domésticas es la parte emocional que va ligada al trabajo de cuidados: es la intersección de estos tres factores lo que configura esta condición (Tereso-Ramírez; Cota, 2017). La doble presencia también se expresa en las constantes indicaciones a pareja o hijos/as para que hagan labores del hogar, de limpieza o de cuidado, debiendo poner atención a sus actividades y a las del resto. Ello puede producir desgaste o conflicto en las relaciones familiares:

Siempre los días que yo voy a trabajar no se levanta [su hija], no se ducha y siempre primero está la pelea de que “¿por qué no te duchaste?” y todo el cuento. La hago ducharse y ordenar su pieza y todas las cosas antes de acostarse... (Karla, comunicación personal, 03.12.2O2O)

(...) pero yo le digo [a su pareja]: "Pero, pucha, lava los platos por lo menos", entonces como que, claro, le dura como... después de las conversaciones le dura una semana de lavar los platos todos los días, y después como que empieza cada 3, después cada 5, y después puede pasar otra semana, dos semanas sin lavar... (Erika, comunicación personal, 03.12.2O2O) 


\section{Estrategias de apoyo escolar y proximidad}

A partir de la suspensión de clases en el país, en marzo de 2020, todas las entrevistadas han debido aprender habilidades del ejercicio docente para ayudar a sus hijos/as con tareas escolares, además de estar frecuentemente presentes durante las clases en línea. Gran parte de la organización de las mujeres gira en torno al horario de clases de sus hijos/as, y su espacio de trabajo suele ser un lugar estratégico para supervisar las clases, lo que, si bien puede generar mayores interrupciones en el propio trabajo, implica una supervisión más directa y permanente hacia los hijos/ as: "como tenían que hacer guías, yo los instalaba acá. Tenía que estar encima de ellos, si no, no lo hacían, entonces aquí nos sentábamos todos juntos en el comedor" (Laura, comunicación personal, 10.12.2020); "entonces, él se pone a trabajar ahí, y yo estoy acá, entonces cualquier cosa a veces me dice: 'mamá, no entiendo esto'" (Erika, comunicación personal, 03.12.2020).

Las mujeres desarrollan estrategias que les permiten apoyar a sus hijos/as en sus tareas escolares y dar seguimiento a sus responsabilidades, conocer el material y calendario escolar, estudiar los contenidos entregados por los colegios, entre otros. Si bien para algunas madres estas actividades son relativamente fáciles (por ejemplo, para las profesoras), para otras suponen habilidades desconocidas hasta ahora, lo que implica frustración, ansiedad, malestar: "porque hasta yo tengo que estudiar lo que estaban estudiando para poder apoyar de verdad" (Susana, comunicación personal, 20.01.2021).

El primer periodo que empezamos con esto en la escuela fue super demandante, muy demandante, y la verdad es que yo estaba super agobiada con el tema del colegio, porque sentía que yo no podía ayudarlo bien, no estaba... yo no soy profesora, entonces no podía ni tenía la paciencia para poder ayudarlo. (Pamela, comunicación personal, 28.12.2O20)

Por un lado, las clases en línea se mencionan como algo positivo porque mantienen ocupados a sus hijos/as durante el día, lo que alivia la obligación de entretenerlos. Por otro, las clases muchas veces generan peleas y conflictos con sus hijos/as al intentar explicar los contenidos. Sumado a eso, muchas familias no cuentan con implementos exclusivos para hijos/as, por lo que deben compartir el computador de trabajo, lo que implica que las madres adapten sus horarios de trabajo a la noche, cuando los hijos/as ya no están en clases. 


\section{Estrategias laborales frente a la sobrecarga}

Como se ha mostrado, frente a la situación de sobrecarga laboral y doméstica, las mujeres despliegan una serie de estrategias cotidianas que permiten enfrentar el día a día laboral, en el contexto donde viven, lo que es muy distinto al empleo que realizaban de manera presencial. Las estrategias desarrolladas apelan a la flexibilidad y experiencia de acomodo permanente que se lleva a cabo en el caso de madres trabajadoras, aunque en estas condiciones se vive de manera más intensa y en copresencialidad de otros/as, principalmente sus hijos/as, como también describen Undurraga, Simbürger y Mora (2021), en el caso de trabajadoras del ámbito académico. Otras estrategias identificadas se relacionan con entablar diálogo con hijos e hijas, para que comprendan las responsabilidades laborales de las madres, lo que en ocasiones da resultado. Algunas estrategias específicas en el ámbito del trabajo remunerado se dan en el marco mismo de la organización laboral, que ha debido ser ajustada en esta coexistencia con lo doméstico, lo cual ha incidido en cuestionamientos de las mujeres sobre las exigencias del empleo, las dinámicas, ritmos y formas de trabajo.

Entonces, tuve que decir en el trabajo: "Mira, mis condiciones eran estas y no puedo más porque se me completó el bote”, porque yo estaba... yo me había comprometido con otros temas, tomando en cuenta que mi trabajo era por dos días a la semana y entonces ahí chantaron la moto conmigo [se ríe], ahí dijeron: "Ya, ya". (Laura, comunicación personal, 10.12.2O2O)

Es que de repente me piden hacer cosas y yo les digo: "Pucha, yo no te lo puedo hacer... [no te lo puedo] entregar antes de un mes", porque trato de ponerme 3 cosas o 4 cosas diarias, porque más de eso no puedo hacer, porque además entre la casa con los niños... (Erika, comunicación personal, 03.12.2O2O)

En el caso de entrevistadas con equipos a su cargo, algunas organizan el trabajo de otra manera, para permitir una mejor coordinación de actividades remuneradas y de cuidados.

Yo conversé con todos los equipos del departamento y les dije que en la hora de colación no podía ser como era antes, que solo es una hora de colación... porque ahora no solo tenías que almorzar, sino que además tenías que preparar el almuerzo, antes tu comprabas el almuerzo hecho, almorzabas en una hora y después podías volver a trabajar, así que el horario de colación en mi departamento se estableció de una a tres de la tarde. (Susana, comunicación personal, 20.01.2021) 


\section{¿Estrategias de resistencia?}

Como parte del análisis, se encuentran algunas estrategias que serán nombradas de resistencia, en el contexto del teletrabajo de las madres. Como se ha señalado, el sistema de género adjudica el papel de cuidados a las mujeres, lo cual exacerba las desigualdades de género -entre otras-frente a este tema. Como se ha visto, estas diferencias se profundizan en el contexto de la pandemia, lo que impacta a las madres de niños/as pequeños/as en términos materiales, espaciales, temporales y afectivos, entre otros. En este escenario, pareciera haber menos posibilidad de agencia, sin embargo, se encuentran modalidades de resistencias cotidianas a nivel micro, que refieren a acciones que llevan a cabo las mujeres, para subvertir o cuestionar, al menos en algunos tiempos y espacios, los mandatos de género en torno a los cuidados y la maternidad, así como el mandato de la productividad sin fin.

Como plantea Haraway (1988), las mujeres, en tanto sujetos, interiorizan los discursos ideológicos dominantes sobre los roles de género y la maternidad, pero, al mismo tiempo que estos discursos se internalizan, también se transforman y se resisten, desarrollando significados y conductas diferentes y potencialmente opuestos (Armstrong; Murphy, 2011). Estas resistencias pueden tomar forma de pensamientos y acciones en la vida cotidiana que, aunque no confrontan directamente, tienen el potencial de comprometerse, negociar y redistribuir o transformar discursos poderosos y provocar un reordenamiento de las relaciones de poder (Hollander; Einwohner, 2004); o bien pueden tomar forma de acciones y movimientos colectivos, transformándose en sujetos políticos.

Desde esta perspectiva, se reconocen estrategias de resistencia de las mujeres, frente a los mandatos de género, de la maternidad, del ser buena madre, buena esposa, responsable del hogar y su familia. Estas estrategias son más o menos explícitas y reconocibles por ellas, pero permiten un espacio de subjetividad que rompe, en cierta medida, con la obligatoriedad de las actividades cotidianas y permite dar espacios de respiro a las mujeres.

Una actividad importante, aunque limitada y por ello valiosa para las mujeres, es la posibilidad de salir del espacio del hogar. Aunque hay permanentes restricciones a la movilidad, algunas entrevistadas aprovechan los permisos que pueden pedir, en el contexto del Plan paso a paso ${ }^{9}$, para hacer algún trámite, pero, además, para despejarse y hacer frente al agobio.

9. El Plan paso a paso es una estrategia gradual para enfrentar la pandemia según la situación sanitaria de cada zona en particular. Se trata de 4 escenarios o pasos graduales, que van desde la cuarentena hasta la apertura inicial, con restricciones y obligaciones específicas. El avance o retroceso de un paso particular a otro está sujeta a indicadores epidemiológicos, red asistencial y trazabilidad ("Paso a paso...”, 2021). 
También yo creo que es importante salir, pero a lo mejor no gastarse ese permiso, cuando hay cuarentena, o no gastarse esa posibilidad de salir en... mejor salir a otra cosa, no sé, a caminar, ¿cachai?, o a juntarte con alguien y no gastarte ese permiso o esa posibilidad de salir. (Natalia, comunicación personal, 28.12.2020)

En términos de los tiempos, el manejo de este puede ser un acto de resistencia (Wearing, 1990), como se ha desarrollado en algunos estudios que indagan en el tiempo de ocio como una práctica de resistencia en madres primerizas frente a la ideología dominante sobre la maternidad. El tiempo libre no tiene el mismo significado entre géneros, ni siquiera entre mujeres con hijos/as y mujeres sin hijos/as. El manejo del tiempo es un recurso estratégico de las madres para dejar momentos para ellas, sin la obligación de hacer actividades para otros, ajustando el ritmo acelerado del día y permitiendo un tiempo de calma y soledad.

Pero como que me despierto más temprano, con el sol, no sé, y ahora me estoy despertando como a las 6:30 [interrumpe su hija para preguntarle algo], me levanto temprano porque como que siento que ese es el rato para mí, porque ellos se despiertan como a las 8:oo, entonces tengo ese rato para bañarme tranquila, me tomo mi café tranquila, veo estas cosas como de redes sociales o me pongo... o cualquier cosa, a veces sacarse las cejas, ese es como el rato de la mañana. (Erika, comunicación personal, 03.12.2O2O)

Un aspecto relevante, se refiere a las estrategias de ajuste en las formas de funcionamiento familiar y redistribución de tareas domésticas que se ha desarrollado en algunos hogares a partir de las medidas de confinamiento. Estos cambios se han dado de manera fácil en algunos casos, mientras en otros ha implicado negociaciones y conflictos. Con el tiempo, dichas modificaciones podrían impactar en el reordenamiento de las dinámicas domésticas entre los géneros y generaciones.

Eso también nos sirvió, en todo caso, porque me tuvieron que empezar a ayudar, tuvieron que empezar a lavar la loza, nos turnábamos. Además, me ayudaban a cocinar. Ya, yo cocino, el otro prepara las ensaladas, el otro guarda la loza que no había secado o guardado de la vez anterior y otro pone la mesa, porque si no yo ahí ya habría colapsado también. (Lucía, comunicación personal, 10.12.2O2O)

La verdad yo me despreocupo totalmente y mi marido hace todo. Él a veces me hace unas preguntas por WhatsApp como: ¿qué hago?, ¿qué cocino? o ¿viste esto?, jajaja; esas cosas, pero son superpocas, así que no, yo casi me despreocupo totalmente, yo me dedico solo al trabajo. (Samanta, comunicación personal, 29.12.2O20)

Las trabajadoras han ido haciendo cambios a partir de la reflexión, conciencia y crítica frente al contexto y a sí mismas, cuestionando las altas exigencias ajenas 
y propias, en los distintos ámbitos de la vida laboral y las rutinas cotidianas. Frente a ello, algunas asumen cambios de actitud y reordenan prioridades. No ha sido un proceso fácil y ello se ha dado, en algunos casos, luego de períodos críticos en las rutinas familiares o individuales, que demostraban la imposibilidad de cumplir con los mandatos en todos los frentes.

Uno de los impactos invisibilizados y poco analizados de la crisis sociosanitaria hace referencia a las consecuencias de los cambios en la salud mental, las cuales han afectado fuertemente a las mujeres. En este aspecto, las madres reconocen problemas de distinta índole e intensidad, que han enfrentado a partir de rutinas cotidianas, pero también buscando apoyo en profesionales especializados en estos temas, sobre todo en el caso de mujeres con mayores recursos.

Tomé estos últimos meses otra actitud, es como... y como que también, por ejemplo, si estoy cansada, me siento no más, po, porque como que antes como que seguía haciendo... se cumple lo que se puede no más, esa es como mi meta, o sea, como mi frase, que se haga lo que se pueda no más. (Erika, comunicación personal, 03.12.2O2O)

Empecé a hacer yoga por un tema de la espalda, pero también me sirvió como terapia emocional y era algo que no podía faltar en el día, de hecho, hasta el día de hoy trato de mantenerme todos los días con algún tipo de ejercicio porque eso también me alivia el tema de la mente, me despeja, ando de mejor ánimo y era como el tiempo que me podía dar. (Carola, comunicación personal, 06.01.2021)

Aunque de manera limitada por las condiciones y medidas sanitarias, el vínculo con otros y el mantenimiento de las redes familiares y de amistad han sido una estrategia de resistencia importante para enfrentar la sobrecarga de trabajo, el miedo, la incertidumbre y la monotonía: "empezamos todos los fines de semana a juntarnos en un departamento, en el otro, hacíamos comidas del mundo, inventar para no enloquecer no más, inventar cosas lúdicas y cantar y, no sé, lo que sea" (Natalia, comunicación personal, 28.12.2020).

Las mujeres desarrollan algunas resistencias en relación con las condiciones laborales y la intensificación de sus actividades. Ello les permite cuestionar la presión, a veces extrema, de los empleadores, que no consideran límites de actividades ni tampoco la condición de cuarentena y de aumento de tareas domésticas, particularmente en el caso de las madres. En ese marco, algunas trabajadoras lo expresan en el espacio laboral, o acomodan sus actividades de manera más realista a las posibilidades de cumplir los objetivos propuestos y las exigencias laborales.

Es que como que yo creo que eso, este período es como que las planificaciones ya no sirven, entonces yo creo que eso también he aprendido ahora, a como: "Ya", o sea, claro, 
trato de tenerme una organización y todo, pero también si la cuestión no resulta, ya filo, no resultó no más, po, sigo igual [se ríe]. (Erika, comunicación personal, 03.12.2O2O)

\section{Conclusiones}

A lo largo del artículo, se indaga sobre las actividades de cuidado (Carrasco, 2013; Batthyány, 2004; 2015), estrategias de cuidado (Batthyány et al., 2017) y resistencias de las mujeres madres trabajadoras con niños pequeños, ante las medidas de confinamiento y el cambio a la modalidad de teletrabajo. A partir de sus relatos, es posible caracterizar distintos tipos de estrategias, que, aunque se superponen, analíticamente se distinguen en 5 modalidades: de organización ante ausencia de control del tiempo; de multiplicidad y simultaneidad de tareas; de apoyo escolar y proximidad; laborales frente a la sobrecarga, y de resistencia.

La condición de madres y la edad de sus hijos/as inciden en las formas como las medidas sociosanitarias impactan en sus rutinas cotidianas, así como en su salud mental, lo que se refleja en sentimientos de rabia, frustración, angustia, agobio, tristeza, entre otros. El ejercicio de las distintas responsabilidades muestra una imagen agobiante frente a las obligaciones en distintos frentes, a la vez que a la concentración de estas en espacios acotados que han debido ajustar a la nueva situación. Frente a ello, sin embargo, las trabajadoras asumen distintas acciones para hacer frente a las nuevas condiciones.

El cierre de colegios, sumado a las cuarentenas obligatorias y al teletrabajo, es el escenario material e institucional que moldea sus rutinas y responsabilidades. Sin embargo, las mujeres también despliegan una serie de estrategias de resistencia, que, de manera generalmente espontánea, van dando respiro a sus vidas, permitiendo un mejor equilibrio en el contexto de crisis. Se evidencian en este ámbito las actividades de autocuidado que desarrollan. Al mismo tiempo, se observan diferencias entre quienes antes de la pandemia podían acceder a externalizar el cuidado u obtener ayuda de las mujeres de la familia extensa, siendo esta una diferencia importante con relación a las mujeres con mayores ingresos, quienes, a pesar de la pandemia, continuaron teniendo ayuda externa remunerada, lo cual, a su vez, deja el cuidado en manos de mujeres más pobres.

En este contexto, llama la atención la ausencia de políticas y medidas del Gobierno, y de las instituciones escolares, así como de una parte importante de los empleadores, destinadas a relevar a las mujeres o apoyar a las familias en las tareas de cuidados, escolaridad y organización laboral. A pesar de la evidente sobrecarga, se sigue naturalizando e invisibilizando (Hatton, 2017) la labor de cuidado de las madres. 
A partir de estos hallazgos, se evidencia la relevancia del espacio doméstico y las actividades de cuidados como ámbitos de reproducción de las desigualdades de género, lo que enfatiza la vulnerabilidad en la condición de las mujeres y las madres trabajadoras. En este escenario, se vuelve central discutir políticas, en el ámbito estatal y privado, que se orienten a hacerse cargo de los cuidados como una actividad del conjunto de la sociedad.

\section{Referencias}

A partir de marzo de 2022 la asistencia a clases presenciales será obligatoria (11 de noviembre de 2021). Ministerio de Educación. Recuperado de https://www.mineduc.cl/en-marzo-del2022-la-asistencia-a-clases-presenciales-sera-obligatoria/

Armstrong, Nathalie; Murphy, Elizabeth (2011). Conceptualizing Resistance. Health: An Interdisciplinary Journal for the Social Study of Health, Illness and Medicine, 16(3), 314-326. https://doi.org/10.1177/1363459311416832

Batthyány, Karina (2004). Cuidado infantily trabajo: ¿un desafio exclusivamente femenino? Una mirada desde el género y la ciudadanía social. Recuperado de https://www.oitcinterfor.org/ sites/default/files/file_publicacion/trazos_2o.pdf

Batthyány, Karina (2015). Laspoliticasy el cuidado en América Latina: una mirada a las experiencias regionales. Recuperado de https://repositorio.cepal.org//handle/11362/37726

Batthyány, Karina; Genta, Natalia; Scavino, Sol (2017). Análisis de género de las estrategias de cuidado infantil en Uruguay. Cadernos de Pesquisa, 47(163), 292-319. https://doi. org/10.1590/198053143710

Boccardo, Giorgio; Andrade, Valentina (2020). Trabajar en tiempos de pandemia. Trabajadoras $y$ trabajadores de los servicios públicos en Chile. Recuperado de http://pop-umbrella. s3.amazonaws.com/uploads/cdc72295-b9db-4baf-aabo-46461d86bd55_Trabajar_en_ Tiempos_de_Pandemia_Informe_de_Resultados.pdf

Brenes, Lucía (2020). Sobre espacios y tiempos en época de pandemia. Reflexiones, 99(2), 1-8. https://doi.org/10.15517/rr.v99i2.42146

Cadem (2020). El Chile que viene. Diferencias de género frente a la crisis del covid-19. Inédito.

Carrasco, Cristina (2013). El cuidado como eje vertebrador de una nueva economía. Cuadernos de Relaciones Laborales, 31(1), 39-56. https://doi.org/10.5209/rev_CRLA.2013.v31.n1.41627

Carrasquer, Pilar (2013). El redescubrimiento del trabajo de cuidados. Algunas reflexiones desde la sociología. Cuadernos de relaciones laborales, 31(1), 91-113. http://doi.org/10.5209/ rev_CRLA.2013.v31.n1.41633 
Comisión Económica para América Latina y el Caribe (2020). La pandemia del COVID-19 profundiza la crisis de los cuidados en América Latina y el Caribe. Recuperado de https:// repositorio.cepal.org/bitstream/handle/11362/45335/5/S2000261_es.pdf

Del Boca, Daniela; Oggero, Noemi; Profeta, Paola; Rossi, Maria Christina (2020). Women's Work, Housework and Childcare, Before and During Covid-19. IZA Institute of Labor Economics. Recuperado de https://papers.ssrn.com/sol3/papers.cfm?abstract_id=3636638

Federici, Silvia (2009). Sobre el trabajo de cuidado de los mayores y los límites del marxismo. Revista Nueva Sociedad, 256, 45-62. Recuperado de https://static.nuso.org/media/articles/ downloads/4103_1.pdf

Flores, Rodrigo (2009). Observando observadores: una introducción a las técnicas cualitativas de investigación social. Santiago: Ediciones UC.

Giaconi, Carolina Andrea (2021). Mujeres, cuidado y resistencias: prácticas de resistencias de mujeres que cuidan a personas con discapacidad severa [Tesis de maestría]. Universidad de Chile, Santiago, Chile.

Haraway, Donna (1988). Situated Knowledges: The Science Question in Feminism and the Privilege of Partial Perspective. Feminist Studies, 14(3), 575-599. https://doi. org/10.2307/3178066

Hatton, Erin (2017). Mechanisms of Invisibility: Rethinking the Concept of Invisible Work. Work, Employment and Society, 31(2), 336-351. https://doi.org/10.1177/0950017016674894

Hilbrecht, Margo; Shaw, Susan; Johnson, Laura; Andrey, Jean (2013). Remixing Work, Family and Leisure: Teleworkers' Experiences of Everyday Life. New Technology, Work \& Employment, 28, 130-144. https ://doi.org/10.1111/ntwe.12010

Hollander, Jocelyn; Einwohner, Rachel (2004). Conceptualizing Resistance. Sociological Forum, 19(4), 533-554. https://doi.org/10.1007/s11206-004-0694-5

Instituto Nacional de Estadísticas (8 de marzo de 2021). Género y empleo: impacto de la crisis económica por covid-19. Boletín estadístico. Recuperado de https://www.ine.cl/docs/defaultsource/genero/documentos-de-an\%C3\%A1lisis/documentos/g\%C3\%A9nero-y-empleoimpacto-de-la-crisis-econ\% 3 3 B3mica-por-covid19.pdf

Ipsos (2020). Claves 2020. Informe abril: Chile en tiempos de Coronavirus. Recuperado de https://www.ipsos.com/sites/default/files/ct/publication/documents/2O2O-O4/ipsoslt_claves_2O2O_-_abril_covid19.pdf

Izcara, Simón Pedro (2007). Introducción al muestreo. Ciudad de México: Porrúa.

Liderar en tiempos de pandemia: propuestas para un retorno a clases presenciales distinto (9 de julio de 2020).Educación 2O2O. Recuperado de https://www.educacion2O2O.cl/noticias/ liderar-en-tiempos-de-pandemia-propuestas-para-un-retorno-a-clases-presencialesdistinto/ 
Lyttelton, Thomas; Zang, Emma; Musick, Kelly (2020). Gender Differences in Telecommuting and Implications for Inequality at Home and Work. Recuperado de https://ssrn.com/ abstract=3645561. http://dx.doi.org/10.2139/ssrn.3645561

Ministerio de Salud confirma primer caso de coronavirus en Chile (3 de marzo de 2020). Ministerio de Salud. Recuperado de https://www.minsal.cl/ministerio-de-salud-confirmaprimer-caso-de-coronavirus-en-chile/

Moré, Paloma (2020). Cuidados y crisis del coronavirus: el trabajo invisible que sostiene la vida. Revista Española de Sociología, 29(3), 737-745. https://doi.org/10.22325/fes/res.2020.47

Moreno, Neus; Moncada, Salvador; Llorens, Clara; Carrasquer, Pilar (2010). Doble presencia, trabajo doméstico-familiar y asalariado: espacios sociales y tiempos. Instituto Sindical de Trabajo, Ambiente y Salud. Recuperado de https://www.ccoo.cat/salutlaboral/docs/ Calaix\%2OSindical/2011_doblepresenciaysalud.pdf

Organización Internacional del Trabajo (2019). Trabajar en cualquier momento y en cualquier lugar: consecuenciasen el ámbito laboral. Recuperadode https://www.ilo.org/wcmsp5/groups/ public/--americas/--ro-lima/--sro-santiago/documents/publication/wcms_723962.pdf

Palma, Irma; Aceituno, Roberto; Duarte, Fabián; Valenzuela, Patricio; Canales, Manuel; Asún, Rodrigo; Riveros, Antonia (2020). Cuarto informe de primeros resultados desestabilización y reorganización de la vida doméstica durante la crisis del covid-19. Recuperado de http://vidaenpandemia.cl/wp-content/uploads/2020/11/CUARTO-INFORME-DEVIDAENPANDEMIA-DESESTABILIZACION-Y-REORGANIZACION-DE-LA-VIDADOMESTICA-DURANTE-LA-CRISIS-DEL-COVID-19-.pdf

Paso a paso, nos cuidamos (2021). Gobierno de Chile. Recuperado de https://www.gob.cl/ coronavirus/pasoapaso/

Pérez, Carmen; Gálvez, Ana María (2009). Teletrabajo y vida cotidiana: ventajas y dificultades para la conciliación de la vida laboral, personal y familiar. Athenea Digital, 15, 57-79. Recuperado de https://www.redalyc.org/articulo.oa?id=53712929004

Presidente anuncia suspensión de clases y reduce actos públicos (15 de marzo de 2020). Ministerio de Salud. Recuperado de https://www.minsal.cl/presidente-anuncia-suspensionde-clases-y-reduce-actos-publicos/

Presidente Piñera decreta Estado de Excepción Constitucional de Catástrofe en todo el país por 90 días para enfrentar coronavirus: "Cuidémonos entre todos" (18 de marzo de 2020). Prensa Presidencial. Recuperado de https://prensa.presidencia.cl/comunicado. aspx?id=148647

Ramacciotti, Karina (2020). Cuidar en tiempos de pandemia. Descentrada, 4(2), e126. Recuperado de https://ri.conicet.gov.ar/handle/11336/114159 
Ruiz, Paulina; Pullas, Paúl; Parra, Cristián Alberto; Zamora, Ruth (2017). La doble presencia en las trabajadoras femeninas: equilibrio entre el trabajo y la vida familiar. Revista de comunicación de la SEECI, 44, 33-51. http://dx.doi.org/10.15198/seeci.2017.44.33-51

Strauss, Anselm; Corbin, Juliet (2002). Bases de la investigación cualitativa. Técnicas y procedimientos para desarrollar la teoría fundamentada. Medellín: Universidad de Antioquia.

Tereso-Ramírez, Leonor; Cota, Beatriz (2017). La doble presencia de las mujeres: conexiones entre trabajo no remunerado, construcción de afectos-cuidados y trabajo remunerado. Margen, 85, 1-12. Recuperado de http://www.margen.org/suscri/margen85/tereso_85.pdf

Tobío, Constanza (1998). Roles de género y la relación familia-empleo. Asparkía, 9, 21-44. Recuperado de http://www.e-revistes.uji.es/index.php/asparkia/article/view/942

Undurraga, Rosario; Simbürger, Elisabeth; Mora, Claudia (2021). Desborde y desazón versus flexibilidad y concentración: teletrabajo académico y género en tiempos de pandemia. Polis Revista Latinoamericana, 2O(59), 12-38. http://dx.doi.org/10.32735/So7186568/2021-N59-1594

Vasilachis, Irene (2006). La investigación cualitativa. En Estrategias de investigación cualitativa (pp. 23-64). Barcelona: Gedisa.

Wearing, Betsy (1990). Beyond the Ideology of Motherhood: Leisure as Resistance. The Australian and New Zealand Journal of Sociology, 26(1), 36-58. 